\title{
Network Formation Analysis with General Allocation Procedures of the Link Costs
}

\author{
T. Hayashida*, I. Nishizaki and H. Katagiri
}

Department of Artificial Complex Systems Engineering, Graduate School of Engineering, Hiroshima University, Higashi-Hiroshima, Hiroshima, 739-8527, Japan

\begin{abstract}
This paper proposes a mathematical model of network formation with general method of share of the link costs. In some published papers of the network formation, it is assumed that all players pay the same amount of cost for forming or maintaining a link, or a player who offers to form a new link pays all of the link cost. In this paper, a mathematical model of network formation with general allocation procedures of the link cost is constructed, and examine stability of the networks. It is assumed that each link cost is shared by a pair of players connected by the link unequally with a fixed fraction. In this paper, it is indicated that the network with certain structure such as the empty, the complete and a star can be stable.
\end{abstract}

\section{INTRODUCTION}

The structure of a network plays an important role in a society. The social networks are sometimes the means for communication or for the allocation of goods. For example, in a firm the network through which relevant information is shared among the employees may have an important effect on the productivity of the firm. The position of a player in the network may affect not only her/his productivity but also the bargaining power of the player relative to others, and this might be reflected in the design of such organizations. The network is defined as the set of members (players) and links connecting the players [1-5].

The relationship between stability and efficiency of the networks is studied in some published papers about the network formation. For a given network if no new link is formed and any existing links are not deleted, then the network is called a stable network. Jackson and Wolinsky [5] define the stability and efficiency of the networks and study the relationship between them. They show a mathematical model in which some of the empty, the complete and a star networks are stable. Moreover, they indicate that a star network is stable, but it is not always efficient. Dutta and $\mathrm{Mu}-$ tuswami [6] construct a mathematical model of network formation with the rule of payoff allocations. Currarini and Morreli [7] and Mutuswami and Winter [8] indicate that some stable network can be efficient network by permitting the negotiation between the players. However, Bala and Goyal [1] propose another mathematical model leading to a result that a wheel network is stable. Hummon [4] examines dynamic processes of the network formation through some numerical experiments under some conditions of link formation and deletion. The results of the experiments indicate that

*Address correspondence to this author at the Department of Artificial Complex Systems Engineering, Graduate School of Engineering, Hiroshima University, Higashi-Hiroshima, Hiroshima, 739-8527, Japan;

E-mail: hayashida@hiroshima-u.ac.jp the network structures are often determined by the size of population and initial structures. Bloch and Jackson [2] construct a mathematical model with considering that the players make transfers and bargain over the formation of links. Feri [9] studies an evolutionary version of the model of Bala and Goyal [1] with exponential decay of payoffs and shows that a star network is stable. Hojman and Szeidl [10] proposed a mathematical model leading a star network with specific structure named peripheral-sponsored star network to be the unique stable network.

In the literature of the network formation, it is assumed that all players pay the same amount of the cost for forming or maintaining a link or one of the players connected by a link pays all of the link cost. However, in the real world, we often observe that some pairs of the players form new links through negotiation on the share of the link cost. In this paper, we construct a mathematical model with asymmetric link costs for examining such situations. In our model, the share of the link cost is not equal, namely one of the players connected by a link pays a larger amount of the link cost for forming or maintaining the link than the other player. We examine the impact of the fraction of payment on the structure and the stability of networks. In this paper, we construct a mathematical model of the network formation with general allocation procedures of the link costs followed Jackson and Wolinsky [5].

In this paper, two players who are connected by a link (link $\{1,2\}$ ) are called a proposer (player 1) and a responder (player 2), respectively. Because the amounts of the payment of the two players are asymmetric like the mathematical models which one of the two players pays all cost $([1,4])$. In this paper, we construct a mathematical model in which two players pays asymmetry amount of cost for forming or maintaining the corresponding link with a fixed fraction. This model can be interpreted as an extensional models of the network formation models of Bala and Goyal [1] and Jackson and Wolinsky [5]. 
The rest of this paper is organized as follows: Section 2 provides some definitions of the networks. In Section 3, we construct a mathematical model of network formation. Finally, we conclude this paper in Section 4.

\section{THE PRELIMINARIES}

\section{The Networks}

In this subsection, we give some definitions of the network formation model according to the model by Jackson and Wolinsky [5].

The network is expressed as a mathematical directed graph model in which a player and a link are represented as a node and an edge, respectively. Let $n$ be the number of players and $N=\{1,2, \ldots, n\}$ be the set of all players. A subset of $N$ of size $2, i j=\{i, j \mid i, j \in N, j \neq i\}$, is called a link, and let $L \subseteq L^{N}=\{i j \mid \forall i, j \in N, j \neq i\}$ be the set of the links. A network is defined as a pair of the set of players and the set of links as $g=(N, L)$.

For a network $g=(N, L)$ and players $i, j, k \in N, i j \in L$ indicates that the link $i j$ is formed, adversely $i k \notin L$ indicates that the link $i k$ is not formed in the network $g$. The network $g=(N, L)$ is called a complete network if $L=L^{N}$, namely all of possible links are formed. A network $g^{\phi}=(N, \phi)$ is called an empty network, namely no link exists in $g^{\phi}$. The complete and the empty networks are shown in Fig. (1a, b).

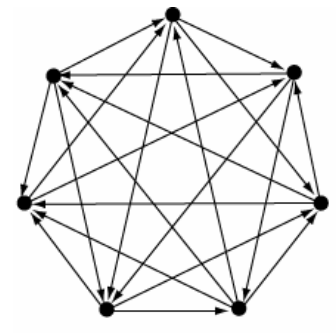

(a) complete network

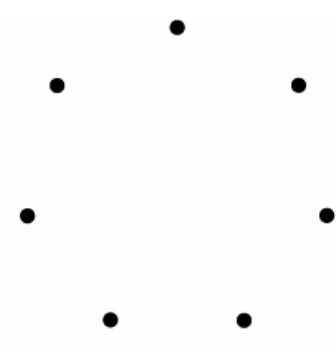

(b) empty network
Fig. (1). Complete network and empty network.

In this paper, for the link $i j \in L, i, j \in N$ in a network $g=(N, L)$, the player $i$ who proposes forming the link $i j$ is called a proposer of the link $i j$, in the same way, the player $j$ on the other side of the link $i j$ is called a responder of the link $i j$. It is assumed that when any two players form new link between them, if the responder accepts the proposal of link formation by the proposer, then the link is formed between them. The network is represented by using mathematical directed graph model as shown in Fig. 1a, b) for discriminating between the proposer and the responder of each link. A player on the origin point of a link is a proposer of the link. A player on the end point of the link is a responder of the link.

For a network $g=(N, L)$ and $\left\{i_{k}, i_{k+1}, i_{k+2}, \ldots, i_{l}\right\} \subseteq N$, a set of links $\left\{i_{k} i_{k+1}, i_{k+1} i_{k+2}, \ldots, i_{l-1} i_{l}\right\} \subseteq L$ is called a path between the players $i_{k}$ and $i_{l}$. If $\left\{i_{k} i_{k+1}, i_{k+1} i_{k+2}, \ldots, i_{l-1} i_{l}\right\} \subseteq L$, then the players $i_{k}$ and $i_{l}$ are said to have a path to each other, and $i_{k} \leftrightarrow^{g} i_{l}$ indicates that the players $i_{k}$ and $i_{l}$ have a path. For a network $g=(N, L), i j \in L$ obviously implies $i \leftrightarrow^{g} j$.
For a network $g=(N, L)$ and a player $i \in N$, let $N_{i}(g)=\{j \mid i j \in L, j \in N, j \neq i\}$ be a set of players who form links with the player $i$. Let $N(g)$ be a set of the players who have at least one link with other players, namely $N(g)=\{i \mid \exists j$ such that $i j \in L\}=\bigcup_{i \in N} N_{i}(g)$. For a network $g=(N, L)$, if the following two conditions hold for the subnetwork $g^{\prime}=\left(N^{\prime}, L^{\prime}\right)$, $N^{\prime} \subseteq N, L^{\prime} \subseteq L$, then the subnetwork $g$ ' is called a component:

(i) For any pair of players $i, j \in N\left(g^{\prime}\right)$ where $i \neq j$, they have a path to each other.

(ii) For any players $i \in N\left(g^{\prime}\right)$ and $j \in N(g)$, if $i j \in L$, then $i j \in L^{\prime}$.

From the above definition, a component is a set the players who have path to each other and the links which are formed by all of them. Therefore, a component in the context of social network theory is a connected component defined in the graph theory.

\section{Utility and Stability of the Networks}

For a network $g=(N, L)$ and a player $i \in N$, let a real valued function $u_{i}(g)$ be a utility function of the player $i$ of the network.

For a network $g=(N, L)$ and players $i, j, k \in N$, supposed that $i j \notin L$ and $i k \in L$. Let $g+i j \equiv(N, L \cup\{i j\})$ be a altered network of $g$ which is formed by addition of the link $i j$, and $g-i j \equiv(N, L \backslash\{i j\})$ be the modified network of $g$ which is formed by deletion of the link $i k$. For any network $g=(N, L)$, when a new link $i j \notin L$ is formed, which is formed by the player $i$ as a proposer for the link and the player $j$ as a responder, let $u_{i, P}^{+i j}(g)$ and $u_{j, R}^{+i j}(g)$ be the increase of utility of the players $i$ and $j$, respectively. An existing link $i k \in L$, which is formed by the player $i$ as a proposer and the player $k$ as a responder is deleted, let $u_{i, P}^{-i k}(g)$ and $u_{k, R}^{-i k}(g)$ be the increment of utility of the player $i$ and $j$, respectively. Then, $u_{i, P}^{+i j}(g), u_{j, R}^{+i j}(g), u_{i, P}^{-i k}(g)$ and $u_{k, R}^{-i k}(g)$ are represented as follows:

$$
\left.\begin{array}{ll}
u_{i, P}^{+i j}(g)=u_{i}(g+i j)-u_{i}(g), & i j \notin L \\
u_{j, R}^{+i j}(g)=u_{j}(g+i j)-u_{j}(g), & i j \notin L
\end{array}\right\}
$$

In this paper, it is assumed that if the utilities of both players increase by forming a link, then the link is formed, and if either player connected by an exiting link increases by deleting the link, then an the link is deleted, according to the connection model by Jackson and Wolinsky. For a network $g=(N, L)$, the network $g$ is a pairwise stable if the following conditions are satisfied [5]: i) $u_{i, P}^{-i j}(g) \leq 0$ and $u_{j, R}^{-i j}(g) \leq 0$, $\forall i, j \in N, \forall i j \in L$; ii) for any $i, j \in N, i j \notin L$, if $u_{i, P}^{+i j}(g) \geq 0$, then $u_{j, R}^{+i j}(g)<0$. In other words, a network is pairwise stable if any existing link is not deleted and no new link is formed in the network. 
Jackson and Wolinsky [5] proposed a mathematical model of network formation in which not only the complete or the empty networks as shown in Fig. (1) but also a star network are pairwise stable. A star network consists of a central player and peripheral players who form links only with the central player. Alternatively, Bala and Goyal [1] proposed a mathematical model with another rule of link formation and deletion in which a wheel network is pairwise stable. Hummon [4] conducted some numerical experiments with several rules of the link formation and deletion, and the result of the experiments indicates that a wheel network can be stable under some other conditions. For example, a star and a wheel networks are shown in Fig. (2a, b).

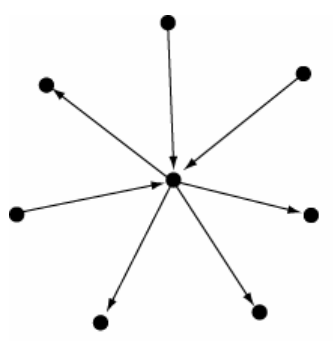

(a) star network

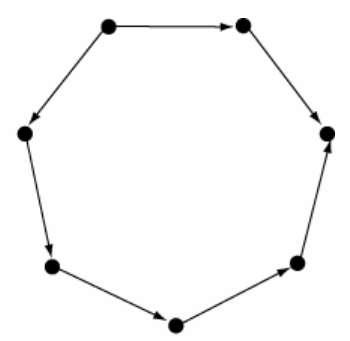

(b) wheel network
Fig. (2). Star network and wheel network.

\section{NETWORK FORMATION}

In some mathematical models of network formation, it is assumed that both players forming a link pay the cost for forming or maintaining the link equally $[4,5]$ or one of the players pays all the cost [1]. These mathematical models lead to the result that the complete, the empty, a star or a wheel networks is the (pairwise) stable. In our mathematical model of network formation, it is assumed that a pair of players connected by a link pays asymmetry amount of cost for the link, namely one of the players pays larger amount of cost for the link than another player connected by a link between them. The network formation model can be interpreted as an extensional model of the existing models by Bala and Goyal [1] and Jackson and Wolinsky [4].

We construct a mathematical model assuming that one of the players connected by a link pays larger amount of cost for the link than another player, namely, in the network formation model, a pair of players share the link cost unequally.

For a network $g=(N, L)$ and players $i, j \in N$, let $t_{i j}(g)$ be a distance between the players $i$ and $j$; the distance is defined as the smallest number of links in the path from $i$ to $j$. Let $\delta \in(0,1]$ be the amount of utility which a player obtains from another player who forms a link with the player. Additionally, let $\pi_{i j}(g)$ be the utility which player $i$ obtains from player $j$ in a network $g$. In this paper, it is assumed that the amount of utility which the player $i$ obtains from the player $j$ depends on the distance between them and $\pi_{i j}(g)=\delta^{t_{i j}(g)}$ if $i \leftrightarrow \leftrightarrow^{g} j$, otherwise $\pi_{i j}(g)=0$ in accordance with the model of Jackson and Wolinsky [5].

For any network $g=(N, L)$ and players $i, j \in N$, let $c_{i j}$ be the link cost for forming or maintaining the link $i j$. We assume that the fraction $\gamma \in[0,1]$ of the cost that of a proposer pays for formation or maintaining a link is fixed. In other words, the proposer of the link $i j$ pays $c_{i j} \gamma$, and the responder pays $c_{i j}(1-\gamma)$ for forming or maintaining the link $i j$. Especially, in case of $\gamma=0.5$, our model coincides with the model of Jackson and Wolinsky [5], and in case of $\gamma=0$, our model coincides with the model of Bala and Goyal [1]. In the model of Jackson and Wolinsky, both players forming a link pay $c$, equally. Namely, the sum of the costs of two players for forming or maintaining each link is $2 c$. According to the setting, in this paper, we assume that $c_{i j}=2 c, \forall i, j \in N$. The utility function of the player $i \in N$ consists of the utilities from other players and link costs, and it is represented as follows:

$$
u_{i}(g)=\sum_{j: i \leftrightarrow^{g} j} \delta^{t_{i j}(g)}-\left(\sum_{N_{i, P}(g)} 2 c \gamma+\sum_{N_{i, R}(g)}\{2 c(1-\gamma)\}\right) .
$$

For a given network $g=(N, L)$ and players $i, j, k, l \in N$ where $i j \notin L, k l \in L$, there exists integers $\alpha_{2}^{y}, \alpha_{3}^{y}, \ldots, \alpha_{n-1}^{y}, y=i, j, k, l$ and the increment of the utilities of players $i, j, k$ and $l$ are represented as follows:

$$
\left.\begin{array}{l}
u_{i, P}^{+i j}(g)=\left(\delta+\alpha_{2}^{i} \delta^{2}+\alpha_{3}^{i} \delta^{3}+\cdots+\alpha_{n-1}^{i} \delta^{n-1}\right)-2 c \gamma, \\
u_{j, R}^{+i j}(g) \\
=\left(\delta+\alpha_{2}^{j} \delta^{2}+\alpha_{3}^{j} \delta^{3}+\cdots+\alpha_{n-1}^{j} \delta^{n-1}\right)-2 c(1-\gamma), \\
u_{k, P}^{-k l}(g)=-\left(\delta+\alpha_{2}^{k} \delta^{2}+\alpha_{3}^{k} \delta^{3}+\cdots+\alpha_{n-1}^{k} \delta^{n-1}\right)+2 c \gamma, \\
u_{k, R}^{-k l}(g)= \\
-\left(\delta+\alpha_{2}^{l} \delta^{2}+\alpha_{3}^{l} \delta^{3}+\cdots+\alpha_{n-1}^{k} \delta^{n-1}\right)+2 c(1-\gamma) .
\end{array}\right\}
$$

Lemma 1 For a given network $g=(N, L)$ and players $i, j, k, l \in N$ where $i j \notin L, k l \in L$,

$$
\left.\begin{array}{l}
u_{i, P}^{+i j}(g) \geq \delta-\delta^{2}-2 c \gamma, \\
u_{j, R}^{+i j}(g) \geq \delta-\delta^{2}-2 c(1-\gamma), \\
u_{k, P}^{-k l}(g) \leq-\delta+\delta^{2}+2 c \gamma, \\
u_{k, R}^{-k l}(g) \leq-\delta+\delta^{2}+2 c(1-\gamma) .
\end{array}\right\}
$$

Proof: For a given network $g=(N, L)$ and $i, j, k, l, m \in N$, $i j \notin L, k l \in L$, in case that a new link $i j$ is formed, the length of the shortest path between players $i$ and $m$ does not increase, and $\delta^{t_{i m}(g+i j)} \geq \delta^{t_{i n}(g)}$ because $\delta \in(0,1]$. For the increment of utility which player $i$ obtains from player $j$, one finds that $\delta^{t_{i m}(g+i j)}-\delta^{t_{i m}(g)}=\delta-\delta^{t_{i m}(g)} \geq \delta-\delta^{2}$ from $t_{i j}(g+i j)=1 \quad$ and $t_{i j}(g) \geq 2$. Because $\delta+m_{2}^{i} \delta^{2}+m_{3}^{i} \delta^{3}+\cdots=\sum_{m \in N: m \neq i}\left(\delta^{t_{m}(g+i j)}-\delta^{t_{i m}(g)}\right)$ $\geq \delta-\delta^{2}$. The condition (6) holds. Similarly, from $t_{k l}(g)=1$ and $t_{k l}(g-k l) \geq 2$, one finds $\delta^{t_{k}(g-k l)}-\delta^{t_{k}(g)} \leq-\delta+\delta^{2}$. Therefore, equation (7) holds.

From Lemma 1, We reveal characteristics of the increment of the utility of the players when a new link is formed or an existing link is deleted. 


\section{Pairwise Stable Networks}

Lemma 2 Let the initial network be the empty network and all the players make decisions in random order. Then once a link formed, it never be deleted.

Proof: For any network $g=(N, L), i j \notin L$, from equations (1) and (2),

$$
\left.\begin{array}{l}
u_{i, P}^{+i j}(g) \geq 0 \Rightarrow u_{i, P}^{-i j}(g+i j) \leq 0, \\
u_{j, R}^{+i j}(g) \geq 0 \Rightarrow u_{j, R}^{-i j}(g+i j) \leq 0 .
\end{array}\right\}
$$

For given two networks $g_{1}=\left(N, L_{1}\right), g_{2}=\left(N, L_{2}\right)$ and a given set of links $L^{\prime} \subseteq L^{N}$, let $L_{2}=L_{1} \cup L^{\prime}$ where $L_{1} \cap L^{\prime}=\phi$. From the proof of Lemma 1, for any players $i, j \in N, i j \notin L_{1}$, $L_{2}, t_{i j}(g+i j) \geq t_{i j}(g)$ and $t_{i j}\left(g_{2}\right) \leq t_{i j}\left(g_{1}\right)$ hold. Namely, the shortest path between any two players in the network $g_{2}$ is shorter than that in the network $g_{1}$. Therefore, from equation (4),

$u_{i, P}^{+i j}\left(g_{2}\right) \geq u_{i, P}^{+i j}\left(g_{1}\right), u_{j, R}^{+i j}\left(g_{2}\right) \geq u_{j, R}^{+i j}\left(g_{1}\right)$.

Similarly, for any players $k, l \in N, k l \in L_{1}, L_{2}$, from equation (5),

$u_{k, P}^{-k l}\left(g_{2}\right) \leq u_{k, P}^{-k l}\left(g_{1}\right), u_{l, R}^{-k l}\left(g_{2}\right) \leq u_{l, R}^{-k l}\left(g_{1}\right)$.

From equation (8), a link which has been formed once in a network never be deleted.

Proposition 1 Let the initial network be the empty network. Then for any pair of c, $\gamma$, a pairwise stable network is formed.

We obtain following theorem for pairwise stable networks. Proofs of Proposition 1 and Theorem 1 are shown in Appendix.

Theorem 1 Let the initial network be the empty network. Then we obtain the followings:

(i) The complete network is uniquely pairwise stable, if

$\gamma<\frac{1}{2}, c \leq \frac{\delta-\delta^{2}}{2-2 \gamma}$, or $\gamma \geq \frac{1}{2}, c \leq \frac{\delta-\delta^{2}}{2 \gamma}$

(ii) A star network is pairwise stable, if

$\gamma<\frac{1}{2}, \frac{\delta-\delta^{2}}{2-2 \gamma}<c \leq \frac{\delta}{2-2 \gamma}$, or

$\gamma \geq \frac{1}{2}, \frac{\delta-\delta^{2}}{2 \gamma}<c \leq \frac{\delta}{2 \gamma}$

(iii) The empty network is uniquely pairwise stable, if

$\gamma<\frac{1}{2}, c<\frac{\delta-\delta^{2}}{2-2 \gamma}$, or $\gamma \geq \frac{1}{2}, c<\frac{\delta-\delta^{2}}{2 \gamma}$

Proposition 1, (i) and (iii) of Theorem 1 indicate that if the utility function of the players are given as the equation (3) and the equation (11) or (13) is satisfied, then either the empty network or the complete network is the unique pairwise stable network. However, if the equation (12) is satisfied, then a star network is pairwise stable but other net- works can be formed and be pairwise stable. The structure of pairwise stable networks corresponding to the pair of the value of the parameters of the link cost $c$ and the fraction of the unequally cost sharing $\gamma$ in Fig. (3). In the figure, the horizontal and vertical axes indicate $c$ and $\gamma$, respectively. Note that it describes in case with $\delta-\delta^{2}<\delta / 2$.

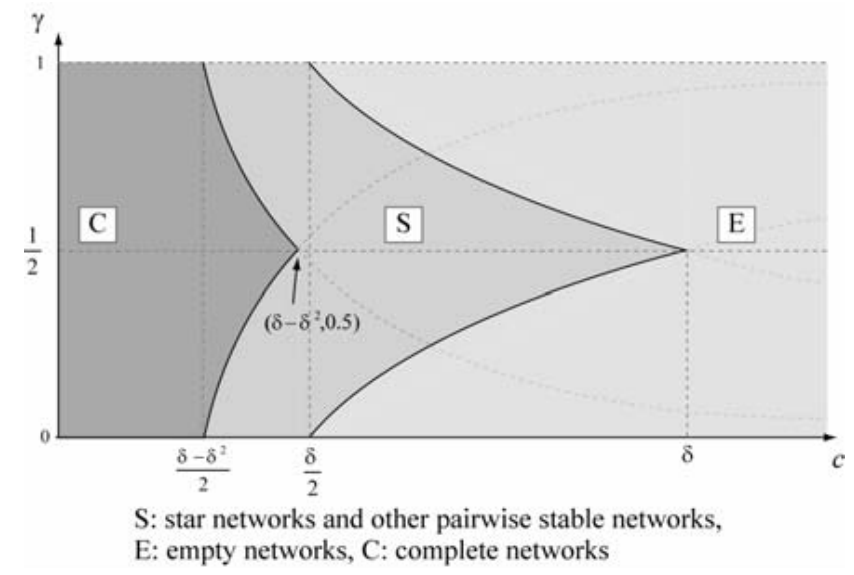

Fig. (3). The structures of pairwise stable networks.

\section{Interpretations}

Note that the complete network consists of larger number of links than a star network, and a star network consists of larger number of links than the empty network. From Theorem 1 and Fig. (3), the number of links is the largest with $\gamma=0.5$. Especially, in case of $\gamma=0.5$ the result of Theorem 1 is consistent with the result of the model of Jackson and Wolinsky [5], and in case of $\gamma=1$ or $\gamma=0$ the result of Theorem corresponds to the result of the model of Bala and Goyal [1]. Therefore, the mathematical model of network formation in which the utility function of players is represented as equation (3) can be interpreted as an extension model of Bala and Goyal [1] and Jackson and Wolinsky [5]. In the region "S" in Fig. (3), a star network is pairwise stable but other structures can be pairwise stable as Jackson and Wolinsky [5] noted.

The fraction of the amount of which each proposer of a link pays for each link approximates to $\gamma=1$ or $\gamma=0$ leads one of the players connected by a link to pay almost all link cost for forming or maintaining, and the utility of the player decreases. Thus, the number of links in a network decreases.

\section{CONCLUSION}

In this paper, we have constructed a mathematical model of network formation with asymmetric link costs. In our model, the share of the cost of a link by two players connected by the player is not equal. In other words, one player pays larger for forming or maintaining the link than another player. In case that the fraction of sharing is particular value, the result of our model is consistent with the models of Jackson and Wolinsky [5] such that two players connected by a link pay same amounts of the cost for the link, and of Bala and Goyal [1] such that one of the players conneceted by a link pays all of the link cost. Therefore, our model of net- 
work formation can be interpreted as an extensional model of these models of network formation.

The simulation analysis using the artificial adaptive agents can be one of the future issues of our network formation models.

\section{APPENDIX}

\section{Proof of Proposition 1}

For a given network $g=(N, L)$ and players $i, j \in N$ where $i j \notin L$, let $b_{i, P}^{+i j}(g)$ and $b_{j, R}^{+i j}(g)$ denote the smallest $c$ s which satisfy $u_{i, P}^{+i j}(g) \leq 0$ and $u_{j, R}^{+i j}(g) \leq 0$, respectively. Therefore, if $c \leq b_{i, P}^{+i j}(g)$ then player $i$ proposes formation of link $i j$. Additionally, if $c \leq b_{j, R}^{+i j}(g)$ then player $j$ accepts the proposal and link $i j$ is formed. Conversely, if $c>b_{i, P}^{+i j}(g)$ or $c>b_{j, R}^{+i j}(g)$ then link $i j$ is not formed.

From equation (5), $b_{i, p}^{+i j}(g)=\left(\delta+m_{2}^{i} \delta^{2}+\cdots\right) / 2 \gamma$. For the empty network, $b_{i, P}^{+i j}\left(g^{\phi}\right)=\delta / 2 \gamma, \forall i, j \in N$, and for a network $g^{N}-i j, \quad b_{i, P}^{+i j}\left(g^{N}-i j\right)=\left(\delta-\delta^{2}\right) / 2 \gamma, \forall i, j \in N$. From proof of Lemma 2, for a given network $g=(N, L)$, $b_{i, P}^{+i j}\left(g^{N}-i j\right) \leq b_{i, P}^{+i j}(g) \leq b_{i, P}^{+i j}\left(g^{\phi}\right)$.

From equation $(5), b_{j, R}^{+i j}(g)=\left(\delta+m_{2}^{i} \delta^{2}+\cdots\right) /(2-2 \gamma)$. For the empty network, $b_{j, R}^{+i j}\left(g^{\phi}\right)=\delta /(2-2 \gamma), \forall i, j \in N$, and for a network $g^{N}-i j, \quad b_{j, R}^{+i j}\left(g^{N}-i j\right)=\left(\delta-\delta^{2}\right) /(2-2 \gamma), \forall i, j \in N$. Similarly, $b_{j, R}^{+i j}\left(g^{N}-i j\right) \leq b_{j, R}^{+i j}(g) \leq b_{j, R}^{+i j}\left(g^{\phi}\right)$ holds.

From the above observation, if the utility function of a player is represented as equation (12), then a star or other network $g=(N, L)$ is formed. If $c>b_{i, P}^{+i j}(g)$ or $c>b_{j, R}^{+i j}(g)$, $\forall i, j \in N, \forall i j \notin L$, then no new link is formed and any existing link is not deleted from Lemma 2. Sequentially, the network $g$ is a pairwise stable network. From Theorem 1 (i) and (iii) as described below, if equation (11) or (13) is satisfied, then the complete or the empty network is a pairwise stable network. Thus, for any set of $(c, \gamma)$, a pairwise stable network is formed.

\section{Proof of Theorem 1}

(i). For a given network $g=(N, L)$ and players $i, j \in N$ where $i j \notin L$, there exists integers $m_{2}^{i}, m_{2}^{j}, m_{3}^{i}, m_{3}^{j}, \ldots$ satisfying $u_{i, P}^{+i j}(g)=\left(\delta+\alpha_{2}^{i} \delta^{2}+\alpha_{3}^{i} \delta^{3} \quad+\cdots \alpha_{n-1}^{i} \delta^{n-1}\right) \quad-2 c \gamma \quad$ and $\quad u_{j, R}^{+i j}(g)=$ $\left(\delta+\alpha_{2}^{j} \delta^{2}+\alpha_{3}^{j} \delta^{3}+\cdots \alpha_{n-1}^{j} \delta^{n-1}\right)-2 c(1-\gamma)$. From Lemma 1, if the equation (11) holds, then $u_{i, P}^{+i j}(g) \geq-2 c \gamma+\delta-\delta^{2} \geq 0$ and $u_{j, R}^{+i j}(g) \geq-2 c(1-\gamma)+\delta-\delta^{2} \geq 0$. Therefore, for a given network $g=(N, L), i j \notin L$, there exists $j \in P_{i}(g)$ and the complete network is formed.

For the complete network $g=\left(N, L^{N}\right), i, j \in N$, if the equation (11) holds, then $u_{i, P}^{-i j}(g)=-\delta+\delta^{2}+2 c \gamma \leq 0$ and $u_{j, R}^{-i j}(g)=-\delta+\delta^{2}+2 c(1-\gamma) \leq 0$. Thus, any link in the complete network never been deleted, the complete network is a pairwise stable network. (ii). For the empty network $g^{\phi}=(N, \phi)$, if the equation (12) is satisfied, $u_{i, P}^{+i j}(g) \geq 0$ and $u_{j, R}^{+i j}(g) \geq 0, \forall i, j \in N$ and there exists a player $k \in P_{i}(g)$, thus a new link $i k$ is formed.

For a star network $g=(N, L)$ which consists of $m$ peripheral players and a single central player, if the equation (12) is satisfied, then for a player $(m+1)$ satisfying $g_{(m+1)}=\phi$, $u_{i, P}^{+i(m+1)}(g)=\delta-2 c \gamma \geq 0 \quad$ and $\quad u_{(m+1), P}^{+(m+1)}(g)=u_{i, P}^{+i(m+1)}(g)=\delta-2 c \gamma \geq 0$. Thus $P_{i}(g)=\{m+1\}, i \in P_{(m+1)}(g)$. Similarly, $u_{(m+1) R}^{+i(m+1)}(g)=\delta$ $+m \delta^{2}-2 c(1-\gamma) \geq 0$ and $u_{i, R}^{+(m+1) i}(g)=\delta-2 c(1-\gamma) \geq 0$. Thus, a new link $i(m+1)$ is formed.

From the above observation, if the equation (12) holds, then a star network is formed. Here, when a new link $i(m+1)$ is formed, there are two kinds of link formation, one is by proposal by a peripheral player $(m+1)$, and one is by proposal by the central player. These two kinds of link formation are described in Figs. (4) and (5).

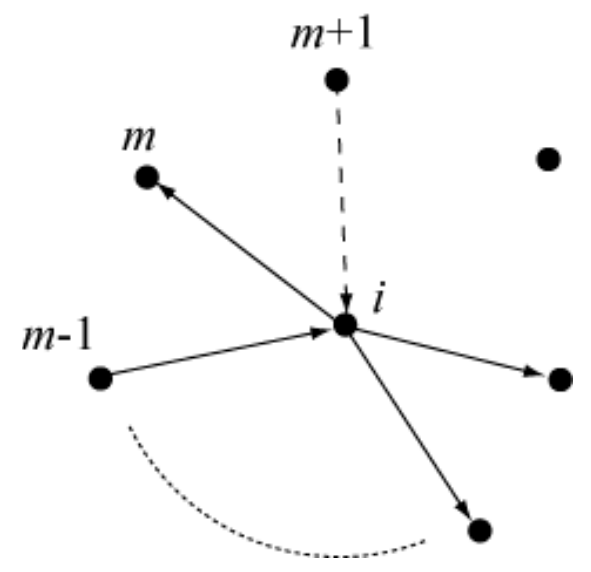

Fig. (4). Proposal by peripheral player $(m+1)$.

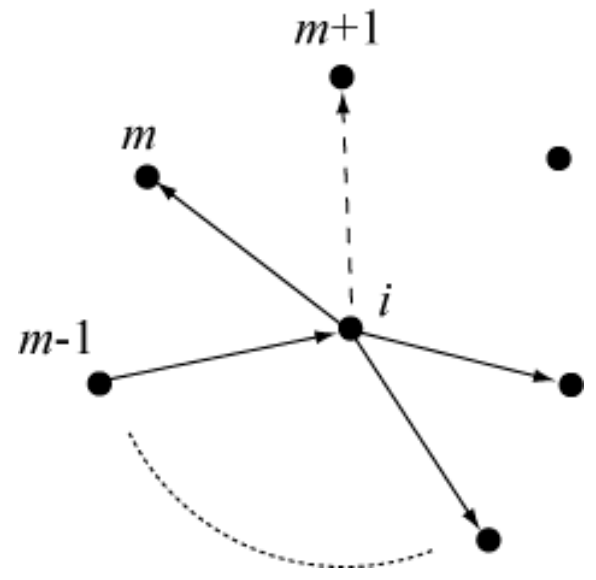

Fig. (5). Proposal by central player $i$,

For a star network $g=(N, L)$ in which player $i$ is the central player, if the equation (12) is satisfied, then $u_{i, P}^{-i j}(g) \geq 0, u_{j, R}^{-i j}(g) \geq 0, \quad \forall j \in N, \quad \forall i j \in L, \quad u_{k, P}^{+k l}(g)$ $=\delta-\delta^{2}-2 c \gamma<0, u_{l, R}^{+k l}(g)=\delta-\delta^{2}-2 c(1-\gamma)<0, \forall k, l \in N$, $\forall k l \notin L$. 
For peripheral players $k, l \in N$ of a star network $g=(N, L)$, if the equation (12) is satisfied, then $u_{j, P}^{+j k}(g)=$ $\delta-\delta^{2}-2 c \gamma \leq 0$, and $u_{k, R}^{+j k}(g)=\delta-\delta^{2}-2 c(1-\gamma) \leq 0$. Thus, a star network is a pairwise star network.

(iii). For the empty network $g^{\phi}=(N, \phi)$, if the equation (13) is satisfied, then $u_{i, P}^{+i j}(g)<0$ and $u_{j, R}^{+i j}(g)<0, \forall i, j \in N$. Thus no new link is formed, and the empty network is the unique pairwise stable network.

\section{REFERENCES}

[1] V. Bala and S. Goyal, "A noncooperative model of network formation," Econometrica, vol. 68, pp. 1181-1229, 2000.

[2] F. Bloch and M. Jackson, "The formation of networks with transfers among players," J. Econ. Theory, vol. 133, pp. 83-110, 2007.
[3] L.C. Freeman, "Social networks and the structure experiment". Research Methods in Social Network Analysis, in L.C. Freeman, D.R. White and A.K. Romney, Eds., Firefax, VA: George Mason University Press, 1989, pp. 11-40.

[4] N.P. Hummon, "Utility and dynamic social network," Soc. Netw., vol. 22, pp. 221-249, 2000.

[5] M.O. Jackson and A. Wolinsky "A Strategic Model of Social and Economic Networks," J. Econ. Theory, vol. 71, pp. 44-74, 1996.

[6] B. Dutta and S. Mutuswami, "Stable networks," J. Econ. Theory, vol. 76, pp. 322-344, 1997.

[7] S. Currarini and M. Morelli "Network formation with sequential demands," Rev. Econ. Des., vol. 5, pp. 229-250, 2000

[8] S. Mutuswami and E. Winter, "Subscription mechanisms for network formation," J. Econ. Theory, vol. 106, pp. 242-264, 2002

[9] F. Feri, "Stochastic stability in networks with decay," J. Econ. Theory, vol. 135, pp. 442-457, 2007.

[10] D.A. Hojman and A. Szeidl, "Core and periphery in networks," $J$ Econ. Theory, vol. 139, pp. 295-309, 2008.

(C) Hayashida et al.; Licensee Bentham Open.

This is an open access article licensed under the terms of the Creative Commons Attribution Non-Commercial License (http://creativecommons.org/licenses/by$\mathrm{nc} / 3.0 /$ ) which permits unrestricted, non-commercial use, distribution and reproduction in any medium, provided the work is properly cited. 